\title{
Simple Motion Evasion Differential Game of Many Pursuers and One Evader with Integral Constraints on Control Functions of Players
}

\author{
Gafurjan Ibragimov and Yusra Salleh \\ INSPEM and Department of Mathematics, UPM, 43400 Serdang, Malaysia \\ Correspondence should be addressed to Gafurjan Ibragimov, gafur@science.upm.edu.my
}

Received 7 May 2012; Revised 15 September 2012; Accepted 15 September 2012

Academic Editor: Alexander Timokha

Copyright (C) 2012 G. Ibragimov and Y. Salleh. This is an open access article distributed under the Creative Commons Attribution License, which permits unrestricted use, distribution, and reproduction in any medium, provided the original work is properly cited.

We consider an evasion differential game of many pursuers and one evader with integral constraints in the plane. The game is described by simple equations. Each component of the control functions of players is subjected to integral constraint. Evasion is said to be possible if the state of the evader does not coincide with that of any pursuer. Strategy of the evader is constructed based on controls of the pursuers with lag. A sufficient condition of evasion from many pursuers is obtained and an illustrative example is provided.

\section{Introduction}

\subsection{Related Works}

Among a large number of papers devoted to differential games with integral constraints (see, e.g., [1-14]), a few works are dedicated to evasion games with integral constraints (see, e.g., $[1,4,7,12])$. Note that integral constraints arise if we consider constraints on energy, resources, and so forth. In [1], an evasion differential game was considered. In the paper [1], the dynamics of the object $z(t)$ is given by the equation

$$
\dot{z}=A z-u+v, \quad z(0)=z_{0},
$$

where $z \in \mathbb{R}^{n}, A$ is a constant matrix, and $u$ and $v$ are control parameters of the pursuer and evader, respectively. The control functions $u(t)$ and $v(t)$ of the pursuer and evader,respectively, are defined as measurable functions satisfying conditions

$$
\int_{0}^{\infty}|u(t)|^{2} d t \leq \rho^{2}, \quad \int_{0}^{\infty}|v(t)|^{2} d t \leq \sigma^{2}
$$


The terminal set $M$ is a subspace of $\mathbb{R}^{n}$. By definition evasion is possible if $z(t) \notin M, t \geq 0$. In this work Pontryagin's method was extended to the evasion game with integral constraints and sufficient conditions of evasion from any initial position were obtained.

In [4], a differential game described by (1.1) was studied. The control functions $u(t)$ and $v(t)$ satisfy conditions (1.2). Evasion is said to be possible if $z(t) \notin M$, where $M=\cup_{i=1}^{m} M_{i}$, $M_{i}$ are subspaces of $\mathbb{R}^{n}$. The pursuers' purpose is to take the point $z(t)$ onto the terminal set $M$; the evader's one, to guarantee evasion from the set $M$. By definition, $l$-escape is possible if $|z(t)| \geq l(t)>0, t \geq 0$ for some function $l(t)$. Sufficient conditions for $l$-escape were obtained.

In the book [12] (chapter 11), linear evasion differential game with integral constraints was examined. The game is described by the equation

$$
\dot{z}=A z-B u+C v, \quad z(0)=z_{0}
$$

where $z \in \mathbb{R}^{n}, A, B$, and $C$ are constant matrices and $u$ and $v$ are control parameters of the pursuer and evader, respectively. The control functions $u(t)$ and $v(t)$ of the pursuer and evader are subjected to the conditions (1.2). The terminal set $M$ is a subspace of $\mathbb{R}^{n}$. By definition evasion is possible if $z(t) \notin M, t \geq 0$. Some sufficient conditions of evasion were obtained.

In [5], a differential game of countably many pursuers and one evader is described by the following infinite system of differential equations:

$$
\dot{z}_{i k}=-\lambda_{k} z_{i k}-u_{i k}+v_{k}, \quad z_{i k}\left(t_{0}\right)=z_{i k}^{0}, \quad k=1,2, \ldots,
$$

which was studied in the Hilbert space $l_{r+1}^{2}$, where

$$
z_{i k}, u_{i k}, v_{k}, t_{0}, z_{i k}^{0} \in \mathbb{R}, \quad z_{i}^{0}=\left(z_{i 1}^{0}, z_{i 2}^{0}, \ldots\right) \in l_{r+1}^{2}, \quad z_{i}^{0} \neq 0
$$

$u_{i}=\left(u_{i 1}, u_{i 2}, \ldots\right)$ is the control parameter of $i$ th pursuer, $i=1,2, \ldots$, and $v=\left(v_{1}, v_{2}, \ldots\right)$ is that of the evader. The control functions of the players are subject to integral constraints

$$
\int_{t_{0}}^{T}\left\|u_{i}(t)\right\|^{2} d t \leq \rho_{i}^{2}, \quad \int_{t_{0}}^{T}\|v(t)\|^{2} d t \leq \sigma^{2}, \quad\|v(t)\|=\left(\sum_{k=1}^{\infty} \lambda_{k}^{r} v_{k}^{2}(t)\right)^{1 / 2}, \quad T>0 .
$$

Avoidance of contact is said to be possible in the game with the initial position $z^{0}=$ $\left\{z_{1}^{0}, z_{2}^{0}, \ldots, z_{m}^{0}, \ldots\right\}, z_{i}^{0} \in l_{r+1}^{2}$, if $z_{i}(t)=\left(z_{i 1}(t), z_{i 2}(t), \ldots\right) \neq 0$, for all $t \in[0, T]$ and $i=1,2, \ldots$ It was proven that if $\sigma^{2}>\sum_{k=1}^{\infty} \rho_{k}^{2}$, then avoidance of contact is possible in the game from any initial position $z^{0}=\left\{z_{1}^{0}, \ldots, z_{m}^{0}, \ldots\right\}, z_{i}^{0} \in l_{r+1}^{2}, z_{i}^{0} \neq 0, i=1,2, \ldots$

The work [7] deals with the evasion differential game of $m$ pursuers $x_{1}, \ldots, x_{m}$ and one evader $y$ described by equations

$$
\dot{x}_{i}=u_{i}, \quad x_{i}(0)=x_{i 0}, \quad \dot{y}=v, \quad y(0)=y_{0}, \quad x_{i 0} \neq y_{0}, \quad i=1, \ldots, m
$$


The game occurs in the plane and the following conditions:

$$
\int_{0}^{\infty}\left|u_{i}(s)\right|^{2} d s \leq \rho_{i}^{2}, \quad i=1, \ldots, m, \quad \int_{0}^{\infty}|v(s)|^{2} d s \leq \sigma^{2}
$$

are imposed on control functions of the players. The main result of the paper is as follow: if $\rho_{1}^{2}+\rho_{2}^{2}+\cdots+\rho_{m}^{2} \leq \sigma^{2}$, then evasion is possible. The important point to note here is the fact that if $\rho_{1}^{2}+\rho_{2}^{2}+\cdots+\rho_{m}^{2}>\sigma^{2}$, then pursuit can be completed [13].

It should be noted that in all of these works, an integral constraint was imposed on the whole control function of each player, such as

$$
\int_{0}^{\infty}|v(s)|^{2} d s \leq \sigma^{2}
$$

However, in contrast to other works, in the present paper, we study a differential game with integral constraints imposed on each coordinate of control functions (see the constraints (1.12)-(1.13)).

At first glance, the inequalities $\rho_{1 i}^{2}+\rho_{2 i}^{2}+\cdots+\rho_{m i}^{2}>\sigma_{i}^{2}, i=1,2$ seem to be the conditions for completing pursuit. It turns out, as shown in the example, evasion is possible from some initial positions even though these inequalities hold.

\subsection{Statement of the Problem}

In $\mathbb{R}^{2}$, we consider an evasion differential game of many pursuers $x_{1}, \ldots, x_{m}$ and one evader $y$ described by the equations

$$
\begin{gathered}
\dot{x}_{i}=u_{i}, \quad x_{i}(0)=x_{i 0}, \quad i=1, \ldots, m, \\
\dot{y}=v, \quad y(0)=y_{0},
\end{gathered}
$$

and assume that $x_{i}^{0} \neq y^{0}, x_{i}^{0}=\left(x_{i 1}^{0}, x_{i 2}^{0}\right), i=1, \ldots, m, y^{0}=\left(y_{1}^{0}, y_{2}^{0}\right)$.

Definition 1.1. A measurable function $u_{i}(t)=\left(u_{i 1}(t), u_{i 2}(t)\right), t \geq 0$ is called an admissible control of the pursuer $x_{i}$ if

$$
\int_{0}^{\infty}\left|u_{i 1}(s)\right|^{2} d s \leq \rho_{i 1}^{2}, \quad \int_{0}^{\infty}\left|u_{i 2}(s)\right|^{2} d s \leq \rho_{i 2}^{2},
$$

where $\rho_{i 1}, \rho_{i 2}, i=1, \ldots, m$ are given positive numbers.

Definition 1.2. A measurable function $v(t)=\left(v_{1}(t), v_{2}(t)\right), t \geq 0$ is called an admissible control of the evader $y$ if

$$
\int_{0}^{\infty}\left|v_{1}(s)\right|^{2} d s \leq \sigma_{1}^{2}, \quad \int_{0}^{\infty}\left|v_{2}(s)\right|^{2} d s \leq \sigma_{2}^{2}
$$

where $\sigma_{1}$ and $\sigma_{2}$ are given positive numbers. 
Definition 1.3. A function of the form

$$
V(t)= \begin{cases}0, & 0 \leq t \leq \varepsilon \\ f\left(u_{1}(t-\varepsilon), \ldots, u_{m}(t-\varepsilon)\right), & t>\varepsilon\end{cases}
$$

is called a strategy of the evader, where $\varepsilon$ is a positive number, $f: \mathbb{R}^{2 m} \rightarrow \mathbb{R}^{2}$ is a continuous function, and $u_{1}(t), \ldots, u_{m}(t), t \geq 0$ are admissible controls of the pursuers.

Definition 1.4. We say that evasion is possible from the initial position $\left(x_{1}^{0}, x_{2}^{0}, \ldots, x_{m}^{0}, y^{0}\right)$ in the game (1.10)-(1.13) if there exists a strategy of the evader $V$ such that $x_{i}(t) \neq y(t), t \geq 0$, $i=1, \ldots, m$ for any admissible controls of the pursuers.

We can now state the evasion problem.

Problem 1. Find conditions for all initial positions $\left(x_{1}^{0}, x_{2}^{0}, \ldots, x_{m}^{0}, y^{0}\right)$ and parameters $\sigma_{1}, \sigma_{2}$, $\rho_{i 1}, \rho_{i 2}, i=1, \ldots, m$ which guarantee evasion in the game (1.10)-(1.13).

This is the main problem to be investigated in the present paper. It should be noted that, in the evasion game, the pursuers use arbitrary admissible controls $u_{1}(t), \ldots, u_{m}(t), t \geq$ 0 , and the evader uses a strategy. By Definition 1.3 this strategy is constructed based on values $u_{1}(t-\varepsilon), \ldots, u_{m}(t-\varepsilon)$.

After the proof of the main result (Theorem 2.1) we will give illustrative examples. For the initial positions, which do not satisfy hypotheses of the theorem, we will show that pursuit can be completed. Therefore, at first we have to give a definition for "pursuit can be completed". To this end we need to define strategies of the pursuers.

Definition 1.5. A Borel measurable function $U_{i}(v)=\left(U_{i 1}(v), U_{i 2}(v)\right), U_{i}: \mathbb{R}^{2} \rightarrow \mathbb{R}^{2}$ is called a strategy of the pursuer $x_{i}$ if for any control of the evader $v(t), t \geq 0$ and the inequalities

$$
\int_{0}^{\infty}\left|U_{i 1}(v(s))\right|^{2} d s \leq \rho_{i 1}^{2}, \quad \int_{0}^{\infty}\left|U_{i 2}(v(s))\right|^{2} d s \leq \rho_{i 2}^{2}
$$

hold.

Definition 1.6. We say that pursuit can be completed from the initial position $\left(x_{1}^{0}, x_{2}^{0}, \ldots, x_{m}^{0}, y^{0}\right)$ in the game $(1.10)-(1.13)$ if there exist strategies of the pursuers $U_{i}$, $i=1, \ldots, m$ such that for any admissible control of the evader the equality $x_{i}(\tau)=y(\tau)$ holds at some $i \in\{1, \ldots, m\}$ and $\tau \geq 0$.

Note that in the pursuit game, the pursuers use strategies and the evader uses any admissible control $v(t)$ (see Definition 1.6). According to Definition 1.5 at current time $t$ the pursuers use $v(t)$ to construct their strategies.

\section{Main Result}

The main result of the paper is formulated as follows. 
Theorem 2.1. Assume the following conditions hold.

(1) There exists a subset I of the set $\{1, \ldots, m\}$ such that

$$
\sum_{i \in I} \rho_{i 1}^{2} \leq \sigma_{1}^{2}, \quad \sum_{i \in J} \rho_{i 2}^{2} \leq \sigma_{2}^{2}, \quad J=\{1, \ldots, m\} \backslash I .
$$

(2) $y_{1}^{0} \notin\left[x_{1}^{\prime}, x_{1}^{\prime \prime}\right], y_{2}^{0} \notin\left[x_{2}^{\prime}, x_{2}^{\prime \prime}\right]$, where

$$
x_{1}^{\prime}=\min _{i \in I} x_{i 1}^{0}, \quad x_{1}^{\prime \prime}=\max _{i \in I} x_{i 1}^{0}, \quad x_{2}^{\prime}=\min _{i \in J} x_{i 2}^{0}, \quad x_{2}^{\prime \prime}=\max _{i \in J} x_{i 2}^{0} .
$$

Then evasion is possible in the game (1.10)-(1.13).

Proof. Let $\rho=\left(\sum_{i=1}^{m} \sum_{j=1}^{2} \rho_{i j}^{2}\right)^{1 / 2}$, and let $\varepsilon, \varepsilon<1 /\left(4 \rho^{2}\right) \min \left\{\left(y_{1}^{0}-x_{1}^{\prime \prime}\right)^{2},\left(y_{2}^{0}-x_{2}^{\prime \prime}\right)^{2}\right\}$, be a positive number. We construct a strategy for the evader as follows:

$$
\begin{aligned}
& v_{1}(t)=\frac{y_{1}^{0}-x_{k 1}^{0}}{\left|y_{1}^{0}-x_{k 1}^{0}\right|} \begin{cases}0, & 0 \leq t \leq \varepsilon, \\
\left(\sum_{i \in I} u_{i 1}^{2}(t-\varepsilon)\right)^{1 / 2}, & t>\varepsilon,\end{cases} \\
& v_{2}(t)=\frac{y_{2}^{0}-x_{l 2}^{0}}{\left|y_{2}^{0}-x_{l 2}^{0}\right|} \begin{cases}0, & 0 \leq t \leq \varepsilon, \\
\left(\sum_{i \in J} u_{i 2}^{2}(t-\varepsilon)\right)^{1 / 2}, & t>\varepsilon,\end{cases}
\end{aligned}
$$

where $u_{1}(t), \ldots, u_{m}(t), t \geq 0$ are any admissible controls of the pursuers, and $k \in I$ and $l \in J$ are arbitrary numbers. The pursuers use any admissible controls and the evader uses the strategy (2.3) and (2.4). We'll prove that evasion is possible. We examine the case $y_{1}^{0}>$ $x_{1}^{\prime \prime}, y_{2}^{0}>x_{2}^{\prime \prime}$. Other cases such as

$$
y_{10}>x_{1}^{\prime \prime}, \quad y_{20}<x_{2}^{\prime} ; \quad y_{10}<x_{1}^{\prime}, \quad y_{20}>x_{2}^{\prime \prime} ; \quad y_{10}<x_{1}^{\prime}, \quad y_{20}<x_{2}^{\prime}
$$

can also be considered similarly. It follows from the inequalities $y_{1}^{0}>x_{1}^{\prime \prime}, y_{2}^{0}>x_{2}^{\prime \prime}$ that $y_{1}^{0}>x_{k 1}^{0}$, $y_{2}^{0}>x_{l 2}^{0}$, and so

$$
\frac{y_{1}^{0}-x_{k 1}^{0}}{\left|y_{1}^{0}-x_{k 1}^{0}\right|}=1, \quad \frac{y_{2}^{0}-x_{l 2}^{0}}{\left|y_{2}^{0}-x_{l 2}^{0}\right|}=1
$$

Then (2.3) and (2.4) take the following form:

$$
\begin{aligned}
& v_{1}(t)= \begin{cases}0, & 0 \leq t \leq \varepsilon, \\
\left(\sum_{i \in I} u_{i 1}^{2}(t-\varepsilon)\right)^{1 / 2}, & t>\varepsilon,\end{cases} \\
& v_{2}(t)= \begin{cases}0, & 0 \leq t \leq \varepsilon, \\
\left(\sum_{i \in J} u_{i 2}^{2}(t-\varepsilon)\right)^{1 / 2}, & t>\varepsilon\end{cases}
\end{aligned}
$$


First, we show that evasion is possible on the time interval $[0, \varepsilon]$. Let $t \in[0, \varepsilon]$. Then for any $i \in I$ we have:

$$
\begin{aligned}
y_{1}(t)-x_{i 1}(t) & =y_{1}^{0}+\int_{0}^{t} v_{1}(s) d s-x_{i 1}^{0}-\int_{0}^{t} u_{i 1}(s) d s \\
& \geq y_{1}^{0}-x_{1}^{\prime \prime}-\int_{0}^{t}\left|u_{i 1}(s)\right| d s \geq y_{1}^{0}-x_{1}^{\prime \prime}-\rho \sqrt{t} \\
& \geq y_{1}^{0}-x_{1}^{\prime \prime}-\rho \sqrt{\varepsilon} \geq \frac{1}{2}\left(y_{1}^{0}-x_{1}^{\prime \prime}\right)>0,
\end{aligned}
$$

since by choice of $\varepsilon, \rho \sqrt{\varepsilon}<(1 / 2)\left(y_{1}^{0}-x_{1}^{\prime \prime}\right)$.

We now show that evasion is possible on $(\varepsilon, \infty)$. If $t \in(\varepsilon, \infty)$, then according to (2.7) for any $i \in I$ we have:

$$
\begin{aligned}
y_{1}(t)-x_{i 1}(t) & =y_{1}^{0}+\int_{\varepsilon}^{t} v_{1}(s) d s-x_{i 1}^{0}-\int_{0}^{t} u_{i 1}(s) d s \\
& \geq y_{1}^{0}-x_{1}^{\prime \prime}+\int_{\varepsilon}^{t} \sqrt{\sum_{i \in I} u_{i 1}^{2}(s-\varepsilon)} d s-\int_{0}^{t} u_{i 1}(s) d s \\
& \geq y_{1}^{0}-x_{1}^{\prime \prime}+\int_{0}^{t-\varepsilon} \sqrt{\sum_{i \in I} u_{i 1}^{2}(s)} d s-\left(\int_{0}^{t-\varepsilon}+\int_{t-\varepsilon}^{t}\right)\left|u_{i 1}(s)\right| d s \\
& \geq y_{1}^{0}-x_{1}^{\prime \prime}-\int_{t-\varepsilon}^{t}\left|u_{i 1}(s)\right| d s \geq y_{1}^{0}-x_{1}^{\prime \prime}-\sqrt{\varepsilon} \rho \\
& \geq \frac{1}{2}\left(y_{1}^{0}-x_{1}^{\prime \prime}\right)>0 .
\end{aligned}
$$

Thus, evasion from the pursuers $x_{i}, i \in I$, is possible. Similarly, according to (2.8) for any $j \in J$ we obtain that

$$
y_{2}(t)-x_{i 2}(t) \geq y_{2}^{0}-x_{2}^{\prime \prime}-\sqrt{\varepsilon} \rho \geq \frac{1}{2}\left(y_{2}^{0}-x_{2}^{\prime \prime}\right)>0, \quad t \geq 0
$$

This completes the proof of the theorem. 
Example 2.2. We consider two differential games of two pursuers and one evader described by the equations

$$
\begin{gathered}
\dot{x}_{i}=u_{i}, \quad x_{i}(0)=x_{i}^{0}, \quad \int_{0}^{\infty} u_{i j}^{2}(s) d s \leq \rho_{i j}^{2}, \\
\dot{y}=v, \quad y(0)=y^{0}, \quad \int_{0}^{\infty} v_{j}^{2}(s) d s \leq \sigma_{j}^{2}, \quad i, j=1,2 .
\end{gathered}
$$

(A) In the first differential game, $\rho_{11}^{2}=4, \rho_{12}^{2}=1, \rho_{21}^{2}=1, \rho_{22}^{2}=4, \sigma_{1}^{2}=\sigma_{2}^{2}=1$, and hence the sets $I=\{2\}, J=\{1\}$ satisfy the first hypothesis of the theorem. Initial positions of the players $x_{1}^{0}, x_{2}^{0}$, and $y^{0}$, where $x_{1}^{0} \neq y^{0}, x_{2}^{0} \neq y^{0}$, are assumed to be any points in the plane. It is not difficult to verify that the second hypothesis of the theorem holds, if $x_{21}^{0} \neq y_{1}^{0}$, and $x_{12}^{0} \neq y_{2}^{0}$ since $x_{1}^{\prime}=x_{1}^{\prime \prime}=x_{21}^{0}, x_{2}^{\prime}=x_{2}^{\prime \prime}=x_{12}^{0}$. Therefore, for this case conclusion of the theorem is true and hence from such initial positions evasion is possible. Note that the meaning of the condition $x_{21}^{0} \neq y_{1}^{0}, x_{12}^{0} \neq y_{2}^{0}$ is that the initial position of the evader $y^{0}$ doesn't lie on the horizontal and vertical lines passing through the points $x_{1}^{0}$ and $x_{2}^{0}$, respectively.

We now construct the strategy for the evader, which guarantees the evasion. Let $u_{1}(t), u_{2}(t), t \geq 0$ be any admissible controls of the pursuers. According to (2.3) and (2.4) the strategy of the evader takes the following form:

$$
v_{1}(t)=\left\{\begin{array}{ll}
0, & 0 \leq t \leq \varepsilon, \\
\xi\left|u_{21}(t-\varepsilon)\right|, & t>\varepsilon,
\end{array} \quad v_{2}(t)= \begin{cases}0, & 0 \leq t \leq \varepsilon, \\
\eta\left|u_{12}(t-\varepsilon)\right|, & t>\varepsilon,\end{cases}\right.
$$

where

$$
\xi=\frac{y_{1}^{0}-x_{21}^{0}}{\left|y_{1}^{0}-x_{21}^{0}\right|}, \quad \eta=\frac{y_{2}^{0}-x_{12}^{0}}{\left|y_{2}^{0}-x_{12}^{0}\right|}
$$

For this example, $\rho=\left(\rho_{11}^{2}+\rho_{12}^{2}+\rho_{21}^{2}+\rho_{22}^{2}\right)^{1 / 2}$ and $\varepsilon$ satisfies the following conditions:

$$
\varepsilon<\frac{1}{4 \rho^{2}} \min \left\{\left(y_{1}^{0}-x_{21}^{0}\right)^{2},\left(y_{2}^{0}-x_{12}^{0}\right)^{2}\right\}
$$

According to the theorem if $x_{21}^{0} \neq y_{1}^{0}, x_{12}^{0} \neq y_{2}^{0}$, then the strategy of the evader (2.14) guarantees the evasion.

However, the theorem gives no information if either $x_{21}^{0}=y_{1}^{0}$ or $x_{12}^{0}=y_{2}^{0}$ since the second hypothesis of the theorem is not satisfied for these cases. We'll show that in each of 
these cases pursuit can be completed in the game (2.12) and (2.13) (see Definitions 1.5 and 1.6). In the case $x_{21}^{0}=y_{1}^{0}$, define the strategies of the pursuers as follows:

$$
\begin{gathered}
u_{11}(t)=0, \quad u_{12}(t)=0, \quad t \geq 0, \\
u_{21}(t)=v_{1}(t), \\
u_{22}(t)= \begin{cases}\frac{1}{\theta}\left(y_{2}^{0}-x_{22}^{0}\right)+v_{2}(t), & 0 \leq t \leq \theta, \\
0, & t>\theta,\end{cases}
\end{gathered}
$$

where $\theta=\left|y_{2}^{0}-x_{22}^{0}\right|^{2}$.

In the case $x_{12}^{0}=y_{2}^{0}$, define the strategies of the pursuers as follows:

$$
\begin{aligned}
& u_{21}(t)=0, \quad u_{22}(t)=0, \quad t \geq 0, \\
& u_{11}(t)= \begin{cases}\frac{1}{\theta_{1}}\left(y_{1}^{0}-x_{11}^{0}\right)+v_{1}(t), & 0 \leq t \leq \theta_{1} \\
0, & t>\theta_{1}\end{cases} \\
& u_{12}(t)=v_{2}(t),
\end{aligned}
$$

where $\theta_{1}=\left|y_{1}^{0}-x_{11}^{0}\right|^{2}$.

We consider the case $x_{21}^{0}=y_{1}^{0}$. The case $x_{12}^{0}=y_{2}^{0}$ can be analyzed in a similar fashion. Admissibility of the strategy (2.17) and (2.18) follows from the following relations:

$$
\begin{aligned}
\int_{0}^{\infty}\left|u_{22}(t)\right|^{2} d t & =\int_{0}^{\theta}\left|u_{22}(t)\right|^{2} d t+\int_{\theta}^{\infty}\left|u_{22}(t)\right|^{2} d t \\
& =\int_{0}^{\theta}\left(\frac{1}{\theta}\left(y_{2}^{0}-x_{22}^{0}\right)+v_{2}(t)\right)^{2} d t \\
& =\int_{0}^{\theta}\left(\frac{1}{\theta^{2}}\left|y_{2}^{0}-x_{22}^{0}\right|^{2}+\frac{2}{\theta}\left(y_{2}^{0}-x_{22}^{0}\right) v_{2}(t)+\left|v_{2}(t)\right|^{2}\right) d t \\
& =\frac{1}{\theta}\left|y_{2}^{0}-x_{22}^{0}\right|^{2}+\frac{2}{\theta} \int_{0}^{\theta}\left(y_{2}^{0}-x_{22}^{0}\right) v_{2}(t) d t+\int_{0}^{\theta}\left|v_{2}(t)\right|^{2} d t \\
& \leq 1+\frac{2}{\theta}\left|y_{2}^{0}-x_{22}^{0}\right| \cdot \int_{0}^{\theta}\left|v_{2}(t)\right| d t+\sigma_{2}^{2} \\
& \leq 2+\frac{2}{\theta}\left|y_{2}^{0}-x_{22}^{0}\right| \cdot \sqrt{\int_{0}^{\theta} 1^{2} d t} \sqrt{\int_{0}^{\theta}\left|v_{2}(t)\right|^{2} d t} \\
& \leq 2+\frac{2 \sigma_{2}^{2}}{\sqrt{\theta}}\left|y_{2}^{0}-x_{22}^{0}\right|=4 .
\end{aligned}
$$


Here, we used the Cauchy-Schwartz inequality. Next, we show that $x_{2}(\theta)=y(\theta)$, that is, pursuit will be completed by the pursuer $x_{2}$ at the time $\theta$. We have

$$
x_{21}(t)=x_{21}^{0}+\int_{0}^{t} u_{21}(s) d s=y_{1}^{0}+\int_{0}^{t} v_{1}(s) d s=y_{1}(t)
$$

for all $t \geq 0$. In particular, $x_{21}(\theta)=y_{1}(\theta)$. In addition,

$$
\begin{aligned}
x_{22}(\theta) & =x_{22}^{0}+\int_{0}^{\theta} u_{22}(s) d s=x_{22}^{0}+\int_{0}^{\theta}\left(\frac{1}{\theta}\left(y_{2}^{0}-x_{22}^{0}\right)+v_{2}(s)\right) d s \\
& =y_{2}^{0}+\int_{0}^{\theta} v_{2}(s) d s=y_{2}(\theta) .
\end{aligned}
$$

Hence, $x_{22}(\theta)=y_{2}(\theta)$. Thus $x_{2}(\theta)=y(\theta)$.

(B) Consider an example of a differential game described by (2.12) and (2.13), for which the second hypothesis of the theorem is not satisfied. Let $\rho_{11}^{2}=2, \rho_{12}^{2}=1, \rho_{21}^{2}=$ $2, \rho_{22}^{2}=1, \sigma_{1}^{2}=\sigma_{2}^{2}=2, x_{1}^{0}=(0,-1), x_{2}^{0}=(0,1), y^{0}=(0, a)$, where $-1<a<1$.

Let the strategies of the pursuers be defined by formulas

$$
u_{11}(t)=u_{21}(t)=v_{1}(t), \quad u_{12}(t)=1, \quad u_{22}(t)=-1, \quad t \geq 0
$$

We show that pursuit is completed by the time $t=1$. Indeed, for all $t \geq 0$ we have

$$
x_{i 1}(t)=x_{i 1}^{0}+\int_{0}^{t} u_{i 1}(s) d s=\int_{0}^{t} v_{1}(s) d s=y_{1}(t), \quad i=1,2
$$

What is left is to show that $x_{i 2}(\tau)=y_{2}(\tau)$ for some $i \in\{1,2\}$ and $\tau, 0<\tau \leq 1$.

It follows from the relations

$$
\begin{gathered}
x_{12}(0)<y_{2}(0)<x_{22}(0) \\
x_{12}(1)=x_{12}^{0}+\int_{0}^{1} u_{12}(s) d s=-1+\int_{0}^{1} 1 d s=0 \\
x_{22}(1)=x_{22}^{0}+\int_{0}^{1} u_{22}(s) d s=1+\int_{0}^{1}(-1) d s=0
\end{gathered}
$$

that one of the equalities $x_{12}(\tau)=y(\tau)$ or $x_{22}(\tau)=y(\tau)$ holds at some $\tau, 0 \leq \tau \leq 1$. According to $(2.24), x_{i 1}(\tau)=y_{1}(\tau)$ and therefore one of the equalities $x_{1}(\tau)=y(\tau)$ or $x_{2}(\tau)=y(\tau)$ holds and hence pursuit is completed at $\tau$. Thus, if the initial position of the evader is in the line segment $\left(x_{1}^{0}, x_{2}^{0}\right)$ then pursuit can be completed by the time $t=1$. 


\section{Conclusion}

We have studied a simple motion evasion differential game with integral constraints on control functions of the players. Unlike the traditional integral constraints, in the present paper the integral constraint is imposed on each component of control functions of the players. Under certain conditions, we have proven that the evasion is possible, that is, we have obtained a sufficient condition of evasion. The problem is open for further investigation if the hypotheses of the theorem are not satisfied. Further studies can be done to obtain complete solution for the evasion problem (1.10)-(1.13).

\section{Acknowledgment}

This research was partially supported by the Research Grant (RUGS) of the Universiti Putra Malaysia, no. 05-04-10-1005RU.

\section{References}

[1] A. Y. Azimov, "A linear differential evasion game with integral constraints on the controls," USSR Computational Mathematics and Mathematical Physics, vol. 14, no. 6, pp. 56-65, 1974.

[2] A. A. Azamov and B. Samatov, $\pi$-Strategy. An Elementary Introduction to the Theory of Differential Games, NUU press, Tashkent, Uzbekistan, 2000.

[3] A. A. Chikrii and A. A. Belousov, "On linear differential games with integral constraints," Trudy Instituta Matematiki i Mekhaniki, vol. 15, no. 4, pp. 290-301, 2009 (Russian).

[4] P. B. Gusiatnikov and E. Z. Mohon'ko, “On $1_{\infty}$-escape in a linear many-person differential game with integral constraints," Journal of Applied Mathematics and Mechanics, vol. 44, no. 4, pp. 436-440, 1980.

[5] G. I. Ibragimov and R. M. Hasim, "Pursuit and evasion differential games in Hilbert space," International Game Theory Review, vol. 12, no. 3, pp. 239-251, 2010.

[6] G. I. Ibragimov, A. A. Azamov, and M. Khakestari, "Solution of a linear pursuit-evasion game with integral constraints," ANZIAM Journal, vol. 52, pp. E59-E75, 2010.

[7] G. I. Ibragimov, M. Salimi, and M. Amini, "Evasion from many pursuers in simple motion differential game with integral constraints," European Journal of Operational Research, vol. 218, no. 2, pp. 505-511, 2012.

[8] N. N. Krasovskii, The Theory of Motion Control, Nauka, Moscow, Russia, 1968.

[9] A. V. Mesencev, "A sufficient conditions for evasion in linear games with integral constraints," Doklady Akademii Nauk SSSR, vol. 218, pp. 1021-1023, 1974.

[10] M. S. Nikolskii, "The direct method in linear differential games with integral constraints," in Control Systems, pp. 49-59, IM, IK, SO AN SSSR, 1969.

[11] B. N. Pshenichnyy and Y. N. Onopchuk, "Linear differential games with integral constraints," Akademii Nauk SSSR, Tekhnicheskaya Kibernetika, vol. 1968, no. 1, pp. 13-22, 1968.

[12] N. Y. Satimov and B. B. Rikhsiev, Methods of Solving of Evasion Problems in Mathematical Control Theory, Fan, Tashkent, Uzbekistan, 2000.

[13] N. Y. Satimov, B. B. Rikhsiev, and A. A. Khamdamov, "On a pursuit problem for n person linear differential and discrete games with integral constraints," Mathematics of the USSR-Sbornik, vol. 46, no. 4, pp. 456-469, 1982.

[14] V. N. Ushakov, "Extremal strategies in differential games with integral constraints," Journal of Applied Mathematics and Mechanics, vol. 36, no. 1, pp. 15-23, 1972. 


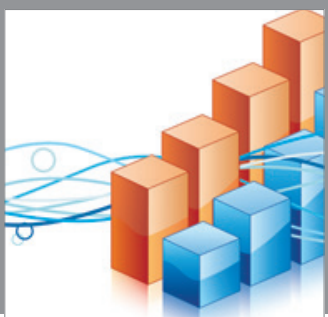

Advances in

Operations Research

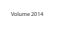

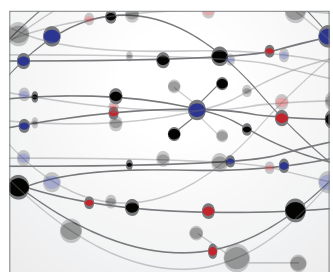

\section{The Scientific} World Journal
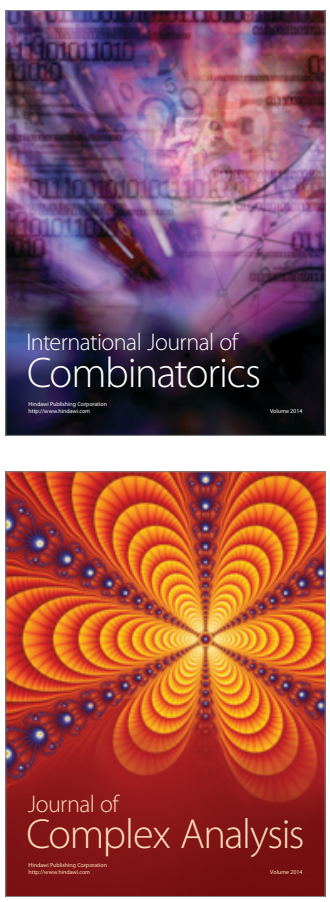

International Journal of

Mathematics and

Mathematical

Sciences
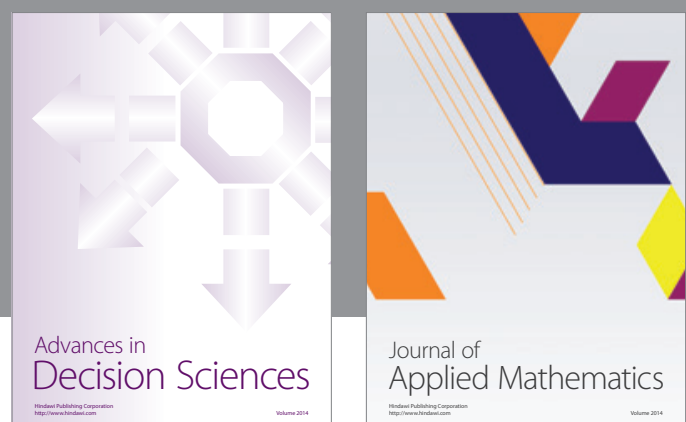

Journal of

Applied Mathematics
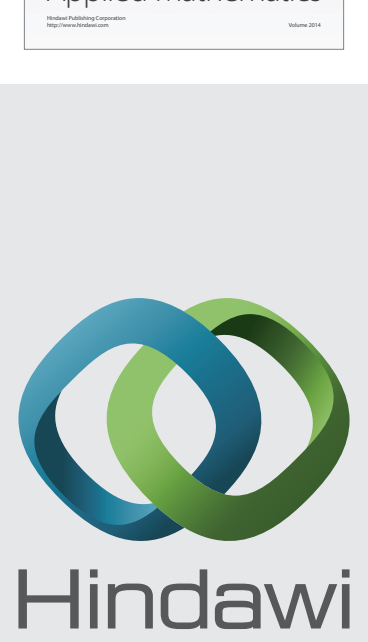

Submit your manuscripts at http://www.hindawi.com
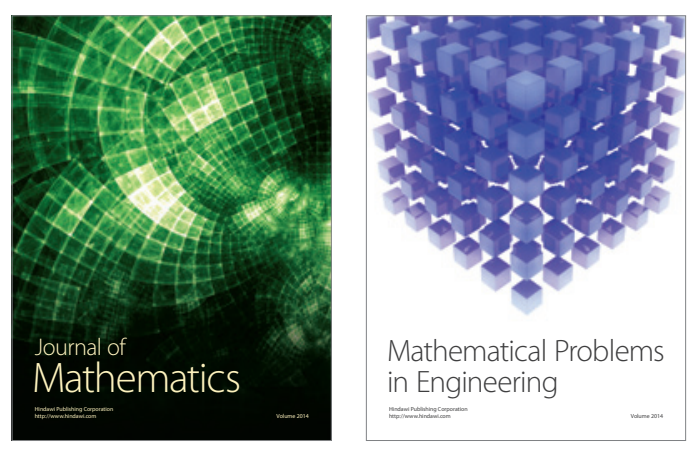

Mathematical Problems in Engineering
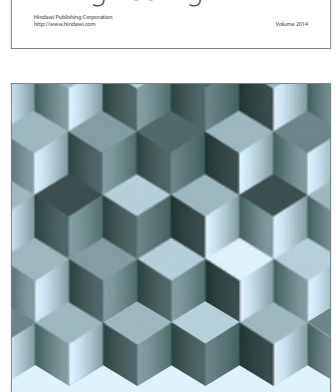

Journal of

Function Spaces
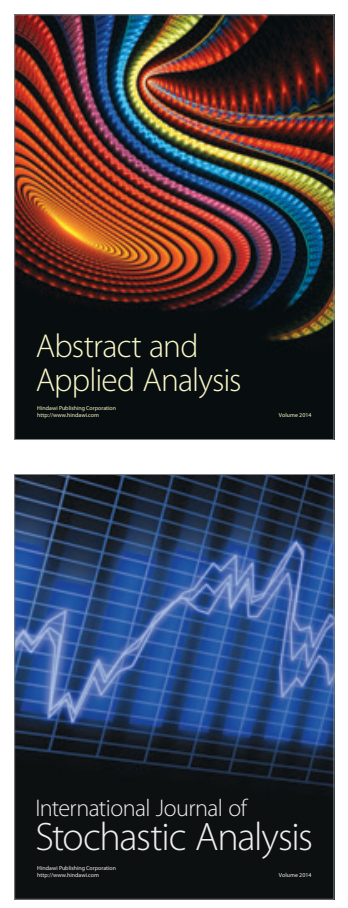

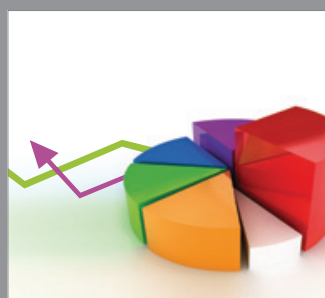

ournal of

Probability and Statistics

Promensencen
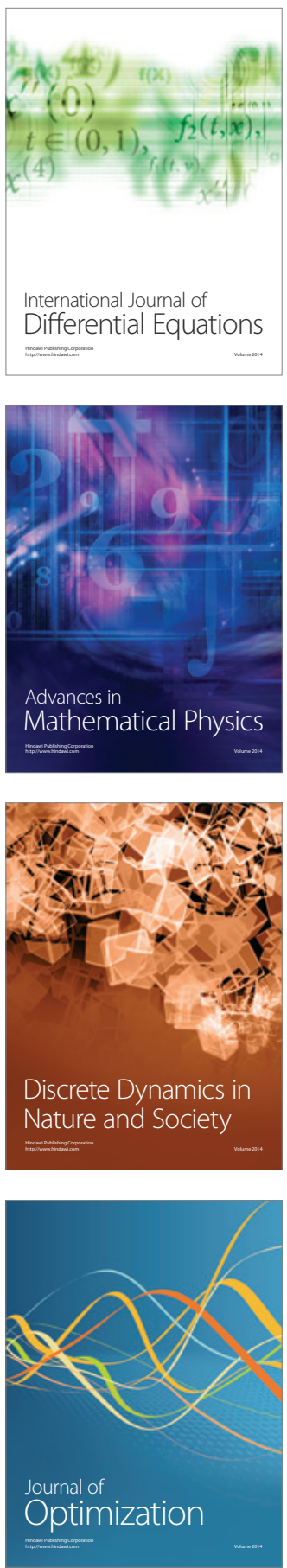